The Astrophysical Journal, in press

\title{
Planetary Nebula Abundances and Morphology: Probing the Chemical Evolution of the Milky Way
}

\author{
Letizia Stanghellini \\ National Optical Astronomy Observatory, 950 N. Cherry Av., Tucson, AZ 85719 \\ lstanghellini@noao.edu \\ Martín Antonio Guerrero \\ Instituto de Astrofísica de Andalucía, Consejo Superior de Investigaciones Cientificas, \\ Apartado Correos 3004, E-18080 Granada, Spain \\ mar@iaa.es \\ Katia Cunha \\ National Optical Astronomy Observatory, 950 N. Cherry Av., Tucson, AZ 85719 \\ kcunha@noao.edu \\ Arturo Manchado \\ Instituto de Astrofísica de Canarias, vía Láctea s/n, La Laguna, E-38200 Tenerife, Spain \\ amt@iac.es \\ Eva Villaver \\ Space Telescope science Institute, 3800 San Martin Drive, Baltimore, MD 21218 \\ villaver@stsci.edu
}

\begin{abstract}
This paper presents a homogeneous study of abundances in a sample of 79 northern galactic planetary nebulae whose morphological classes have been uniformly determined. Ionic abundances and plasma diagnostics were derived from selected optical line strengths in the literature, and elemental abundances were
\end{abstract}


estimated with the Ionization Correction Factor developed by Kingsbourgh \& Barlow (1994). We compare the elemental abundances to the final yields obtained from stellar evolution models of low- and intermediate-mass stars, and we confirm that most Bipolar planetary nebulae have high nitrogen and helium abundance, and are the likely progeny of stars with main-sequence mass larger than $3 \mathrm{M}_{\odot}$. We derive $<\mathrm{Ne} / \mathrm{O}>=0.27$, and discuss the implication of such a high ratio in connection with the solar neon abundance. We determine the galactic gradients of oxygen and neon, and found $\Delta \log (\mathrm{O} / \mathrm{H}) / \Delta \mathrm{R}=-0.01$ dex $\mathrm{kpc}^{-1}$ and $\Delta \log (\mathrm{Ne} / \mathrm{H}) / \Delta \mathrm{R}=-0.01 \mathrm{dex} \mathrm{kpc}^{-1}$. These flat PN gradients do not reconcile with galactic metallicity gradients flattening with time.

Subject headings: Planetary nebulae: general; abundances; chemical evolution of the Milky Way

\section{Introduction}

Planetary nebulae (PNe) are ejected at the tip of the Asymptotic Giant Branch (AGB) evolutionary phase in low and intermediate mass stars, and they have been used as probes of the galactic nucleosynthesis history and stellar evolution in many studies. The PN abundances may be used as probes of primordial nucleosynthesis when studying the elements with zero yields through the AGB evolution (oxygen, neon, and argon) and as probes of stellar evolution through the analysis of the products of the evolutionary nucleosynthesis (helium, nitrogen, and carbon).

One important aspect of PN chemical composition studies is the application to determining of abundance gradients in the galaxy. Metallicity gradients represent one of the most important constraints for models of galaxy formation and evolution. Besides PNe, metallicity gradients can be measured in other galactic populations, ultimately probing distinct time periods covering the formation and evolution of the galactic disk. For the young populations, metallicity gradients are measured using H II regions and OB stars; the intermediate age populations are represented by Cepheid variables, while the oldest populations are probed with studies of open clusters and PNe. A question of significant importance in this context is whether there has been an increase or decrease of the abundance gradients with time.

In this study we present results from a homogeneous abundance analysis for the elements helium, nitrogen, oxygen, neon, and argon in a large sample of PNe and derive galactic metallicity gradients for the elements oxygen and neon. The strength of the present study is multi-fold. First, our PNe are uniformly distributed within the galaxy, with a well-defined 
coverage $\left(0^{\circ}<l<240^{\circ}\right)$; second, the PN distances adopted here are the best available for each nebula; third, the abundances were obtained homogeneously and self-consistently; and last, the morphology of these PNe has been classified in a uniform fashion, and used for stellar population selection. In $\S 2$ we review the morphological classification that has been used, and the choice of PN distances. In $\S 3$ we describe the method used for abundance determination. In $\S 4$ we present the results of our analysis, and we summarize our findings and conclusions in $\S 5$.

\section{Morphology and Distances}

The PN sample used in this study consists of galactic PNe in the northern sky that have a morphological determination. The PN images were selected mainly from the Manchado et al. (1996) catalog, complemented by those PNe from Balick's (1987) and Schwarz et al.'s (1992) samples needed to populate homogeneously the volume of space defined by Manchado et al.'s survey, as described in Manchado et al. (2000). The PN morphological classes used here are described in Stanghellini et al. (2002): Round (R), Elliptical (E), and Bipolar (B) PNe. Bipolar Core (BC) PNe, those nebulae whose main contour is elliptical and that show inner ring structure, are included in the major elliptical class except when explicitly noted. We used this database to explore the chemical evolution of the Milky Way, using morphology as an indication of stellar population.

Several recent studies have determined that PN morphology is a powerful indicator of stellar population. In particular, R PNe may belong to the solar neighborhood (Manchado et al. 2000), and may be the progeny of the lower mass progenitors (Stanghellini et al. 2002). Elliptical PNe are found uniformly through the galactic disk, thus they represent the low to intermediate mass progenitors. Bipolar PNe have typically very low altitudes on the galactic plane, high nitrogen abundances, and possibly even high mass central stars (Stanghellini et al. 1993 and 2002, Corradi \& Schwarz 1995, Manchado et al. 2000). These characteristics seem to conjure that the progenitors of B PNe belong to the higher-end mass range of the AGB stars $\left(\mathrm{M}>3 \mathrm{M}_{\odot}\right)$. Finally, BC PNe show a morphological complex or ring-like structure, but they do not have extended lobes. They are probably a mixed group, with some E with

inner structures and a few B PNe whose lobes are below the surface brightness limits of the observations. From a population viewpoint they may belong either to the E or B groups.

Distances to galactic PNe are very hard to acquire. While precise individual distances, are known only for a small faction of galactic PNe, statistical distance scales are calibrated to allow the derivation of an approximate distance for the rest of the galactic sample. The statistical method by Cahn et al. (1992) is based on the relation between the (inverse) surface 
brightness and the ionized mass of the PNe. Other methods are based on similar relations that hold for PNe on a physical basis, and all statistical distance scales are calibrated with PNe of known distances. In Cahn et al. (1992) the maximum scatter of the distances is $\Delta \mathrm{d} / \mathrm{d} \sim 0.4$. As all statistical distance scales, the Cahn et al. scheme is only accurate as long as one can measure unambiguously the area of the PN projection on the sky plane, and this is not straightforward for B PNe. This fact should be taken into account when using bipolar PN and a statistical distance scale to infer gradients and other distance-dependent quantities.

In this paper we select individual distances from Acker et al. (1992) for the PNe where at least one individual distance was available, and we use their averages in our gradient calculations. In all other cases, we use the statistical distances from Cahn et al. (1992), where available, or those calculated with the same method by Stanghellini et al. (2002). We believe that our distances, both individual averages and statistical, are the best possible available for the PNe in our sample. We have compared our statistical distances to individual averages for the PNe whose individual distances are available, and found a correlation coefficient of 0.6, which made us comfortable to use our statistical distances for the remaining PNe.

\section{Abundances}

In order to overcome the inhomogeneity problem that afflicts most PN abundance compilations we have compiled our own set of relative emission line intensities for the PNe in our sample and analyzed them in a homogeneous fashion.

Most of the relative emission line intensities have been adopted from the Catalog of Relative Emission Line Intensities Observed in Planetary Nebulae (Kaler, Shaw, \& Browning 1997), also available on line, including all high-quality emission line fluxes published before May 1995. We complemented the Kaler et al. (1997) catalog with emission line fluxes from additional, more recent references (Guerrero \& Manchado 1996 for A 58; Kaler et al. 1996 for K 3-51, K 3-94, M 2-52, and M 3-28: Trammell et al. 1993 for CRL 618: Guerrero et al. 1996 for K 4-55; and Cuisinier et al. 1996 for PC 19). In total, we have assembled a database of emission line intensities (emission line intensities relative to $\mathrm{H} \beta$ ) for $19 \mathrm{R}, 45 \mathrm{E}$ (9 of which BC), and 15 B PNe. Overall, in this study we can count on 79 northern galactic PNe with homogeneously derived abundances and morphologies.

The published intensities have then been averaged, uncorrecting for extinction in the few cases where needed, by using the originally published extinction constants. We have used only emission intensities from the PN as a whole, and excluded from averages these 
intensities relative to halos, knots, and fliers. We then corrected the intensity ratio averages for interstellar extinction, using the theoretical intensity ratios by Brocklehurst (1971), and the standard reddening law by Whiteford (1958).

The plasma diagnostic $\left(N_{\mathrm{e}}, T_{\mathrm{e}}\right)$ and ionic chemical abundances have then been computed using the nebular analysis package in IRAF/STSDAS (Shaw et al. 1998). The helium abundances have been computed including the collisional effects described by Clegg (1987). Finally, the elemental abundances have been determined applying the ionization correction factors (ICFs) following the prescription that Kingsburgh \& Barlow (1994, hereafter KB94) give for the case where only optical lines are available.

In Table 1 we give the elemental abundances derived as described above. The first column gives the common PN name, then we give the helium, nitrogen, oxygen, neon, and argon abundances in terms of hydrogen, as $\mathrm{X} / \mathrm{H}$, where $\mathrm{X}$ represents the chemical elements. Note that the helium abundance has been multiplied by $10^{2}$, nitrogen, oxygen, and neon values have been multiplied by $10^{4}$, and argon abundances by $10^{6}$. Averages of all elemental abundances for each morphological type are reported at the end of each morphological group in the Table. We estimate that typical uncertainties are $\sim 10 \%$ for the oxygen, helium, and nitrogen abundances, and 15 to $20 \%$ for the neon and argon abundances, depending on the ionization stages available in the analyzed spectra.

We compare our abundances to large samples of abundances published in recent years. In Table 2 we report the average oxygen abundances and their standard deviation for three PN samples: our own PNe (column [2]); the sample that we have in common with Perinotto et al. (2004, hereafter PMS04), which includes 27 PNe (column [3]); and the sample that we have in common with Henry et al. (2004, hereafter HKB04), which includes 17 PNe (column [4]). In row 1 we give the averaged oxygen abundance from our work, for the three samples respectively. In row 2 we give, in columns 3 and 4, the averaged oxygen abundances using the abundances from PMS04 and HKB04 respectively, and the samples in common with our sample, so that the columns are readily comparable.

Perinotto et al. (2004) calculated abundances from averages of published intensity lines. We have 27 objects in common with their sample, and our average oxygen abundance (and its scatter) compare reasonably well with theirs (column 3, Table 2) for the object in common. By correlating the individual oxygen abundances of PNe in common between our sample and that of PMS04 we obtain a correlation coefficient of 0.5 , and most oxygen abundances in common agree within the uncertainties.

Henry et al. (2004) have determined the abundance of a large sample of PNe from an homogeneous set of observations. Henry et al.'s work overlaps with our morphological 
data-set with 17 PNe in common. Abundances of PNe from ours calculations and HKB04's correlate well (with correlation coefficient of $0.8,0.7$, and 0.6 for $\mathrm{O} / \mathrm{H}, \mathrm{N} / \mathrm{H}$, and $\mathrm{Ne} / \mathrm{H}$ respectively) but the HKB04 oxygen and neon abundances are systematically higher (by a factor of $\sim 1.5$ ) than ours. We attribute this offset to the use of different Ionization Correction Factors (ICF) for oxygen and neon. HKB04 used ICF $(\mathrm{O})=\left(\mathrm{He}^{+}+\mathrm{He}^{++}\right) / \mathrm{He}^{+}$, as described in Kwitter and Henry (2001), while we have used $\operatorname{ICF}(\mathrm{O})=\left(\left(\mathrm{He}^{+}+\mathrm{He}^{++}\right) / \mathrm{He}^{+}\right)^{2 / 3}$, as defined by KB94. The ICF for oxygen abundance is also a scaling factor for the neon ICF, and this explains the scaling of neon abundances as well.

Costa et al. (2004) measured oxygen abundances for a sample of anti-center PNe, also used $\mathrm{ICF}(\mathrm{O})=\left(\mathrm{He}^{+}+\mathrm{He}^{++}\right) / \mathrm{He}^{+}$. We have only six PNe in common with the Costa et al.'s (2004) sample, and we recover an offset between our and their abundances, their oxygen abundances being $\sim 0.18$ dex higher than ours, confirming the origin of the abundance offset using different ICFs. In order to use our neon and oxygen abundances together with another sample of published abundances a re-scaling by the appropriate ICF ratio is advised, if using ICFs different than those of KB94.

\section{Results}

\subsection{Effects of Stellar Evolution}

In Figure 1 we plot the N/O ratio against helium abundances for the PNe in our sample ${ }^{1}$. This is a classical diagnostic plot to discriminate between Type I and non-Type I PNe (Peimbert \& Torres-Peimbert 1983), and to study the connection between high mass PN progenitors and enhanced chemical abundances of helium and nitrogen (Kaler et al. 1990). We have plotted the PNe of our study with different symbols for the different morphological types (hereafter open circles: R; asterisks: E; triangles: BC; squares: B PNe). In Figure 1 we note the high segregation of $\mathrm{B} P N e$ toward the higher helium and N/O.

The N/O ratio is a well-known diagnostic ratio for nucleosynthesis in AGB stars. Nitrogen is produced in AGB stars in two ways: by neutron capture, during the CNO cycle, and by hot-bottom burning, if the base of the convective envelope in AGB stars is hot enough to favor the conversion of ${ }^{12} \mathrm{C}$ to ${ }^{14} \mathrm{~N}$. The hot-bottom burning produces primary nitrogen, contrary to the CNO cycle, that produces secondary carbon and nitrogen. Since hot-bottom burning may occur only in the most massive of the AGB stars (with turnoff mass $\mathrm{M}_{\mathrm{TO}}$ larger than $3 \mathrm{M}_{\odot}$, van der Hoek and Groenewegen 1997), nitrogen is expected to be enriched, with

\footnotetext{
${ }^{1}$ We use the notation $\mathrm{A}(\mathrm{X})=\log \mathrm{X} / \mathrm{H}+12$, thus $\mathrm{A}(\mathrm{N})-\mathrm{A}(\mathrm{O})=\log (\mathrm{N} / \mathrm{O})$.
} 
respect to oxygen, in those $\mathrm{PNe}$ whose progenitors were the most massive. A correlation between nitrogen enrichment and high mass central stars has been observed by Kaler and Jacoby (1990). The helium abundance also depends on the initial mass of the progenitor. Helium is enriched progressively from $\mathrm{M}_{\mathrm{TO}}=1$ to $3 \mathrm{M}_{\odot}$, reaches a plateau between 3 and 4 $\mathrm{M}_{\odot}$, and then increases again toward the higher masses (Marigo 2001).

In this work, we have not defined Type I PNe a priori, but we obtain that morphologically distinct PNe belong to different loci in the plane of Fig. 1. By comparing the data distribution of Fig. 1 to the stellar evolutionary models of Marigo (2001; thick line for $\mathrm{M}_{\mathrm{TO}}>3 \mathrm{M}_{\odot}$, thin line for $\mathrm{M}_{\mathrm{TO}}<3 \mathrm{M}_{\odot}$ ) we see that all the $\mathrm{B}$ PNe are within the domain of the high mass stars, while the R PNe are predominantly in the low-mass domain, while most $\mathrm{E}$ PNe are in the same locus as the R PNe, a few in the B PN locus.

It is worth noting that the yields from theoretical models reflect very specific initial conditions for the elements that do not vary with AGB evolution. For example, the range of $\mathrm{A}(\mathrm{O})$ in Marigo's models $(8.90$ - 9.0) is much more narrow than the observed one $(7.73$ $9.09)$.

\subsection{Oxygen, Neon, and Argon Abundances}

One remarkable result of our abundance analysis is the very tight correlation between neon and oxygen abundances, as plotted in Figure 2. The correlation coefficient between the two abundances is 0.9 for the whole sample. We calculated the average ratio of the neon versus oxygen abundances, obtaining $<\mathrm{Ne} / \mathrm{O}>=0.27 \pm 0.10$ for the whole sample, and, consistently, $<\mathrm{Ne} / \mathrm{O}>=0.27 \pm 0.09$ for $\mathrm{E} \mathrm{PNe}$ alone. The almost identical average of the Ne/O ratios for the whole sample and for Elliptical PNe indicates that neon and oxygen abundances are locked independent of morphology, i.e., independent on stellar population. The relation between neon and oxygen abundances, and its independency on PN population, is a direct consequence of the fact that both neon and oxygen derive from primary nucleosynthesis, mostly in stars with $\mathrm{M}>10 \mathrm{M}_{\odot}$, thus independent on the evolution of the PN progenitors through the AGB. It is worth noting that the same correlation and averages would have been found even if we had used the alternative ICF (e. g. HKB04).

We do not find a correlation between the argon and oxygen (or neon) abundances, with global correlation coefficient of 0.1 . Argon and neon both derive from primary nucleosynthesis, respectively from the carbon and oxygen burning, occurring in Type I but especially

Type II SNe. The nucleosynthesis of argon necessitates the presence of alpha particles, and it is enhanced in presence of secondary elements such as carbon and oxygen in the environment 
(Clayton 2003). This may be the cause for the distinct trends of argon and neon in PNe, or, more likely, the larger uncertainties in the argon abundance calculation hid a possible correlation between the two species.

\subsubsection{Comparison with Solar Ne/O and Implications for Solar Models}

The average $\mathrm{Ne} / \mathrm{O}$ ratio obtained from our PN sample is almost a factor of 2 higher than the most recent assessment of Ne/O in the Sun by Asplund et al. (2005) that recommends a value of $\mathrm{Ne} / \mathrm{O}=0.15$. The solar $\mathrm{Ne} / \mathrm{O}$ ratio, however, is uncertain because the neon abundance in the Sun is not measured from lines that form in the solar photosphere, but from high energy particles in the solar corona. The average Ne/O ratio obtained from our sample is very consistent with an average $\mathrm{Ne} / \mathrm{O}$ computed for the sample analyzed in HKB04 ( $\mathfrak{i N e} / \mathrm{O}_{i}=0.28$ for the PNe in common with our sample). These higher than solar $\mathrm{Ne} / \mathrm{O}$ ratios obtained for PNe, both in this study as in HKB04, are interesting when put into the context of the recent debate about the $\mathrm{Ne} / \mathrm{O}$ ratio in the Sun and implications for solar models.

One of the successes in solar physics has been the good agreement obtained between predictions from solar models and oscillation measurements from helioseismology. Recently, however, the solar abundances of key elements such as, C, N, and O have been revised downward by roughly 0.2-0.3 dex, according to abundance analyses based upon more realistic 3-dimensional hydrodynamical model atmospheres (see Asplund et al. 2005 for a review). Solar models that adopt these revised solar abundances as input can no longer satisfactorily match the helioseismological observations. One way to reconcile the solar models with observations, however, is to adopt a significantly higher neon abundance for the Sun. This possibility has been investigated by Bachall et al. (2005) who concluded that it would suffice to raise the neon abundance in the Sun to 8.29 (or, $\mathrm{Ne} / \mathrm{O}=0.42$ ) in order to bring solar models back into agreement with the helioseismological measurements.

A least-squares fit to the abundances obtained for the $\mathrm{PN}$ in this study yields the following relation for neon versus oxygen: $(\mathrm{Ne} / \mathrm{H})=0.3446(\mathrm{O} / \mathrm{H})-2.348 \times 10^{5}$. If we plug in the solar oxygen abundance value of $\mathrm{A}(\mathrm{O})=8.67$ (Allende Prieto, Lambert, \& Asplund 2000 ) in this relation, we obtain $\mathrm{A}(\mathrm{Ne})=8.14$, which is significantly higher than the currently estimated solar value of $\mathrm{A}(\mathrm{Ne})=7.84$. We argue that the high $\mathrm{Ne} / \mathrm{O}$ obtained for $\mathrm{PNe}$ provides indirect support to a potentially higher neon abundance in the Sun, in comparison with the recommended value by Asplund et al. (2005). This argument is based on the simple fact that a fraction of the PN in our sample evolved from progenitor stars with low masses. In particular, if we consider the Round PNe, which are very likely the progeny of one solar 
mass stars, we obtain an average $\mathrm{Ne} / \mathrm{O}=0.26$. Therefore, the results obtained for $\mathrm{PNe}$ would be more in line with a somewhat higher neon abundance that is needed in order to, at least partially, solve the solar model problem.

\subsection{Galactic Metallicity Gradients}

In order to obtain the elemental gradients we calculate the PN galactocentric distances with the standard formulation (e. g., Maciel \& Lago 2005). We used as input $\mathrm{R}_{\odot}=8.0 \mathrm{kpc}$, the distance to the galactic center, and we used statistical and individual PN distances as described in $\S 2$. Galactocentric distances used here are given in column (7) of Table 1, where we indicate whether the distance is derived from Cahn et al.'s (1992) statistical scheme (superscripts 1 to 4), or is an average of individual distances from Acker et al.'s (1992) (superscript 5). We estimated the random uncertainty of the statistical distances by assuming the maximum relative error of $40 \%$ in the heliocentric distances from Cahn et al. (1992) (see §2), and by assuming that the error in the galactic longitude and latitude of the $\mathrm{PNe}$ is very small in comparison with the distance error. The final relative errors in the galactocentric distances are rarely higher than $60 \%$, the random uncertainty being $20 \%$ on average. In Table 1 we note - with superscripts 1 to 4 - whether the relative distance error is better than $20 \%$, between 20 and $40 \%$, between 40 and $60 \%$, and larger than $60 \%$. These errors only take into account the (maximum) errors from the statistical distance scale, but not the asymmetry of the PN: for B PNe, the uncertainty on the statistical distance may be larger than quoted, depending on the nebular shape. The uncertainties of the individual distances in Table 1 can be found in the original papers, as quoted by Acker et al. (1992). We calculate the spread of individual distances, where more than one distance was available, and found that the different determinations agree to better than $10 \%$ in most cases, with an average spread of about $5 \%$ in the individual distance determinations. Note that individual distances and their averages do not suffer the B PN uncertainty, since they are not based on angular diameter measurements. We did not use halo PNe in the gradient analysis, thus did not enter galactocentric distances for halo PNe in Table 1.

In Figure 3 we show the galactic gradients of the oxygen and neon abundances in the PNe of our sample. In these figures we plot the different morphologies with distinct symbols, as described in Figure 1. We can study these gradients with the added insight of the morphological classes.

Bipolar PNe are the progeny of more massive stars that formed more recently, and since oxygen and neon abundance should not vary during the evolution through AGB and postAGB, their oxygen and neon abundances are probes of relatively young stellar population 
compared with $\mathrm{R}$ and $\mathrm{E}$ PNe. A possible exception is the alternative pathway to neon production in low metallicity 2-4 $\mathrm{M}_{\odot}$ stars (Gibson et al. 2005, and references therein). Unfortunately B PNe are not very useful to establish galactic gradients for two reasons: first, they can not be seen at large distances, due to their proximity to the galactic plane and consequent higher extinction. In Figure 3, for example, B PNe are probes of galactic gradients only between 3 and $9 \mathrm{kpc}$. Second, and most importantly, their statistical distances may also be more uncertain than those of other PNe, since their sizes are not very well defined from the observations. Round PNe may belong to a faint, local population (Manchado et al. 2000). If this is true, their loci on the plots of Figure 3 are deceiving, and they are poor probes of gradients as well. It is best to use $\mathrm{E}$ and $\mathrm{BC}$ PNe for gradient estimates. Ideally, a gradient should be estimated with individual distances only. In out case, about $1 / 3$ of the PNe in our sample have individual distances, thus it represent an improvement with respect to other samples. On the other hand, individual distances to PNe are only available for nearby objects, thus have limited use for galactic gradients. In our sample, individual galactocentric distances are in the $7-9 \mathrm{kpc}$ range.

The oxygen gradient that we obtained including E and BC PNe of Figure 3 (top panel) is very shallow $\left(\Delta \log (\mathrm{O} / \mathrm{H}) / \Delta \mathrm{R}=-0.01 \pm 0.02 \mathrm{dex} \mathrm{kpc}^{-1}\right)$. The neon gradient obtained for the sample of $\mathrm{E}$ and $\mathrm{BC} \mathrm{PNe}$ is the same than the oxygen gradient, as expected from the previous discussion of $\S 4.2$. PNe NGC 2242 and M2-44 have not been included in this gradient, due to large uncertainties in their neon abundances.

The oxygen and neon gradients determined by using the complete PN sample are identical to those above, except that the scatter is reduced to $0.01 \mathrm{dex} \mathrm{kpc}^{-1}$. The scatter in the gradients incorporate the maximum distance scale uncertainties and the abundance errors.

The argon abundance distribution has a slope of $\Delta \log (\mathrm{Ar} / \mathrm{H}) / \Delta \mathrm{R}=-0.05 \mathrm{dex} \mathrm{kpc}^{-1}$ for the $\mathrm{E}$ and $\mathrm{BC} \mathrm{PNe}$, with very large scatter due to the larger uncertainties in the abundance derivation.

\subsubsection{Comparisons with Gradients from the Literature}

Several studies in the literature are devoted to determinations of abundance gradients in selected samples belonging to distinct populations in the galaxy. As discussed in the introduction, these include H II regions, young stars, Cepheids, open clusters and PNe. The abundances that define such gradients are derived from a diverse sets of objects that require very different analysis techniques. This diversity results in different systematic errors in the abundances for the different populations of objects. In addition, measurements of metallicity 
gradients in the galaxy are uncertain due to significant errors in the adopted distances for the individual objects. As a consequence, the magnitudes of the best-fit slopes to the different data-sets of abundances versus galactocentric distances have varied significantly, making in hard to separate the astrophysical variance (due to gradient changes with time, for example) from the uncertainties.

Compilations of elemental gradients obtained for galactic populations can be found in different studies (e.g. Matteucci 2003; Stasinska 2004). In the following we mention results from the most recent studies of gradients in young populations. For early-type stars, Daflon \& Cunha (2004) conducted a homogeneous and self-consistent study of a large sample of OB stars and found much flatter gradients than previous studies of abundances in early-type stars. For oxygen, in particular, they found a relatively flat gradient of $-0.03 \mathrm{dex} \mathrm{kpc}^{-1}$ (neon and argon gradients have not been derived in studies of OB-type stars). For H II regions, Deharveng et al. (2000) and Pilyugin et al. (2003) also found, for oxygen, a flatter gradient $\left(\Delta \log (\mathrm{O} / \mathrm{H}) / \Delta \mathrm{R}=-0.04 \mathrm{dex} \mathrm{kpc}^{-1}\right)$ than previous $\mathrm{H}$ II region studies. In addition, the gradients found recently for neon and argon by Martin-Hernandez et al. (2002) are also relatively flat: $\Delta \log (\mathrm{Ne} / \mathrm{H}) / \Delta \mathrm{R}=-0.04 \operatorname{dex~kpc} c^{-1}$ and $\Delta \log (\mathrm{Ar} / \mathrm{H}) / \Delta \mathrm{R}=-0.05 \operatorname{dex~kpc}^{-1}$.

A recent determination of metallicity gradients based on PNe comes from the work by HKB04. In Table 3 we assemble our gradients for the studied elements oxygen, neon and argon, as well as other $\mathrm{PN}$ results from the literature. It is interesting to note that the abundance gradients derived in HKB04 are slightly flatter than those obtained in Martins \& Viegas (2000, MV00) and Maciel \& Quireza (1999, MQ99). Our oxygen gradient is $\Delta$ $\log (\mathrm{O} / \mathrm{H}) / \Delta \mathrm{R}=-0.01$ dex $\mathrm{kpc}^{-1}$, quite different from the steep value obtained previously by MQ99 (from abundances compiled from the literature); and still significantly flatter than the $\mathrm{O} / \mathrm{H}$ gradient derived in $\mathrm{HKB} 04$. For neon, the situation is similar: our derived gradient is also much flatter than all other studies.

It is worth noting that all distances used by MQ99, as well as by HKB04, derive form Maciel (1984) calculation, and those do not compare as well as our statistical distances to individual distances. Interestingly, if we estimate the galactic gradients from the abundances given in Table 1 and Maciel (1984) distances, we find $\Delta \log (\mathrm{O} / \mathrm{H}) / \Delta \mathrm{R}=-0.04 \mathrm{dex} \mathrm{kpc}^{-1}$ and $\Delta \log (\mathrm{Ne} / \mathrm{H}) / \Delta \mathrm{R}=-0.03$ dex kpc${ }^{-1}$, much closer to HKB04 or MQ99 gradients. It is natural to conclude that the choice of distances can make a difference when estimating galactic gradients from PN abundances.

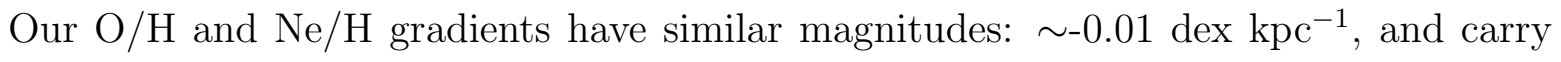
average scatter of about 1 dex. An agreement between oxygen and neon gradients is to be expected from predictions of stellar nucleosynthesis. In principle, the oxygen and neon abundances evolve in lock-step as both of these elements are produced in short lived massive 
stars. Agreement between neon and oxygen gradients, within the uncertainties, was also obtained for the sample of galactic PNe studied by HKB04. A similar result was derived by Crockett et al. (2006) from a study of H II regions in M33. We note, however, that MQ99 do not find agreement between the best-fit slopes for their neon and oxygen PNe data. For argon, our best-fit slope is steeper than the one obtained by HKB04, but it is in agreement with MQ99. The Ar abundances, however, are more uncertain and the obtained abundances scatter over $\mathrm{a} \sim 1.5$ dex interval at roughly galactocentric distances between 6 and $12 \mathrm{kpc}$.

It is important to recognize that metallicity gradients derived for populations of different ages are in fact probing distinct epochs of disk evolution. In this context, Maciel et al. (2003) divided their PN sample in 3 age groups so that possible time variations in the galactic gradients could be investigated. Maciel et al. (2003, 2005) concluded both from their PN sample, from open clusters samples (Friel et al. 2002; Chen et al. 2003) and from comparisons with gradients obtained recently for young stars (Daflon \& Cunha 2004), and HII regions (Deharveng et al. 2000), that there is significant flattening of metallicity gradients with time. The oxygen and neon gradients obtained in this study are flat and very difficult to reconcile with this picture, given that our sample of PNe do not support the existence of steep gradients earlier in the galaxy.

We also determined the gradients for two groups of $\mathrm{PNe}$ with low $(\log \mathrm{N} / \mathrm{O}<-0.5)$ and high $(\log \mathrm{N} / \mathrm{O}>-0.5)$ nitrogen abundance, in order to reproduce the Type I and non-Type I gradients, and found no significant differences between the two types, confirming that our results are not compatible with gradients flattening with time.

Costa et al. (2004) have measured abundances of PNe in the direction of the galactic anti-center, with galactocentric distances between 8 to $14 \mathrm{kpc}$. Although they do not publish the gradient derived by their sample alone, we calculate that their oxygen gradient is $\Delta \log$ $(\mathrm{O} / \mathrm{H}) / \Delta \mathrm{R}=-0.01 \mathrm{dex} \mathrm{kpc}^{-1}$, which is also very flat and agrees with our gradient.

For argon we find, unlike neon and oxygen, a steep gradient. As discussed previously, however, the Ar abundances derived here are the most uncertain and there is a very large scatter in the obtained abundances. In addition, argon abundances and respective gradients are measured in PNe, but not in early-type stars, nor open clusters. Therefore, it is not straightforward to use this element in order to investigate time variations in the gradients.

\section{Conclusions}

In this paper we analyze elemental abundances of helium, oxygen, nitrogen, neon, and argon for a sample of northern galactic PNe from the Manchado et al. (1996) morphological 
catalog, complemented with other PNe in the Schwarz et al.'s (1992) and Balick's (1987) compilation that fit our criteria, to build a volume complete sample. The difference between this and previous studies is that we have used PN morphology as an extra tool to differentiate between the progenitors populations. Our abundances correlate well with previously published samples that were calculated with similar ICFs. The abundance offset between ours and HKB04 (and Costa et al. 2004) oxygen and neon abundances is mostly due to these ICF differences. Future studies should take these differences into account, and work on the comparison between the HKB04 and other abundance determination methods is in progress (Henry and Kwitter, private communication).

We confirm that $\mathrm{B}$ PNe have higher $\mathrm{N} / \mathrm{O}$ ratios and helium abundances than $\mathrm{R}$ and $\mathrm{E}$ $\mathrm{PNe}$, marking the different range of progenitor's initial mass of the B PNe with respect to $\mathrm{R}$ and $\mathrm{E} \mathrm{PNe}$.

We found a tight correlation between $\mathrm{A}(\mathrm{O})$ and $\mathrm{A}(\mathrm{Ne})$, and determined an average ratio of $\langle\mathrm{Ne} / \mathrm{O}\rangle=0.27 \pm 0.10$. We also discussed the implications that such a ratio has on the determination of solar abundances, and review the possible alternatives.

By using the $\mathrm{E}$ and $\mathrm{BC} \mathrm{PNe}$, whose distances are reliable and whose distribution is more uniform in the galaxy we found gradients of the order of $\Delta \log (\mathrm{O} / \mathrm{H}) / \Delta \mathrm{R}=-0.01$ dex $\mathrm{kpc}^{-1}$ compared to previously determined gradients of $-0.04 \mathrm{dex} \mathrm{kpc}^{-1}$. Our gradients are not consistent with a flattening of gradients with time. Our abundances and distances are sound, and the abundances compare well with similarly determined quantities by other groups. We conclude that the galactic abundance gradients are flat, but also that a definite answer will only be possible when more objects at larger and very small galactocentric distances will be detected, since the set of distances used for gradients have a great importance in the results. By selecting PN population in the inner disk one should be careful not to include bulge PNe, belonging to a different population. A different choice of the ICFs, while changing the elemental abundances, would not affect the gradients. Furthermore, the uncertainties in the statistical distances may affect the gradients, and more sets of individual distances would improve the determination. Other classes of objects should be studied in addition of the PNe to extend the gradients to larger distances.

Given that PN distances carry such large uncertainties, we use distance-independent ways to disclose abundance gradients. For example, if we average the oxygen abundance for PNe with $0^{\circ}<l<=45^{\circ}$ and with $135^{\circ}<l<240^{\circ}$ we sample respectively the PNe in the direction of the galactic center $(\mathrm{GC})$ and anti-center $(\mathrm{GA})$. We obtain $<\mathrm{O} / \mathrm{H}>_{\mathrm{GC}}=$ $3.79 \pm 2.26 \times 10^{-4}$ and $<\mathrm{O} / \mathrm{H}>_{\mathrm{GA}}=2.91 \pm 1.23 \times 10^{-4}$, translating into a difference of 0.11 dex in oxygen abundance between the galactic center and the anti-center directions, confirming the rather flat gradient that we derive in the more traditional way in this paper. 
We warmly thank Karen Kwitter, Richard Henry, and Bruce Balick for thoroughly discussing with us the different abundance determination methods, and Verne Smith for scientific discussion. We thank an anonymous Referee for several important suggestion that improved our presentation. 


\section{REFERENCES}

Acker, A., Marcout, J., Ochsenbein, F., Stenholm, B., \& Tylenda, R. 1992, Garching: European Southern Observatory, 1992

Allende Prieto, C., Lambert, D. L. \& Asplund, M. 2001, ApJ, 556, 63

Asplund, M., Grevesse, N. \& Sauval, A. J. 2005, in Cosmic Abundances as Records of Stellar Evolution and Nucleosynthesis, ed. T. G. Barnes III \& F. N. Bash (San Francisco: ASP), ASP conf. Ser., 336, p. 25

Bahcall, J. N., Basu, S., \& Serenelli, A. M. 2005, ApJ, 631, 1281

Balick, B. 1987, AJ, 94, 671

Brocklehurst, M. 1971, MNRAS, 153, 471

Cahn, J. H., Kaler, J. B., \& Stanghellini, L. 1992, A\&AS, 94, 399

Chen, L., Hou, J. L., \& Wang, J. J. 2003, AJ, 125, 1397

Clayton, D. 2003, Handbook of Isotopes in the Cosmos, by Donald Clayton, pp. 326. ISBN 0521823811. Cambridge, UK: Cambridge University Press, October 2003.

Clegg, R. E. S. 1987, MNRAS, 229, P31

Costa, R. D. D., Uchida, M. M. M., \& Maciel, W. J. 2004, A\&A, 423, 199

Corradi, R. L. M., \& Schwarz, H. E. 1995, A\&A, 293, 871

Crockett, N. R., Garnett, D. R., Massey, P., Jacoby, G. 2006, ApJ, 637, 741

Cuisinier, F., Acker, A. \& Köppen J. 1996, BAAS, 307, 215

Daflon, S., \& Cunha, K. 2004, ApJ, 617, 1115

Deharveng, L., Pena, M., Caplan, J., Costero, R. 2000, MNRAS, 311, 329

Friel, E. D., Janes, K. A., Tavarez, M., Scott, J., Katsanis, R., Lotz, J., Hong, L., \& Miller, N. 2002, AJ, 124, 2693

Gibson, B. K., Fenner, Y., \& Kiessling, A. 2005, Nuclear Physics A, 758, 259

Guerrero, M. A., \& Manchado, A. 1996, ApJ, 472, 711

Guerrero, M. A., Manchado, A., \& Serra-Ricart, M. 1996, ApJ, 456, 651 
Henry, R. B. C., Kwitter, K. B., \& Balick, B. 2004, AJ, 127, 2284

Kaler, J. B., Shaw, R. A., \& Kwitter, K. B. 1990, ApJ, 359, 392

Kaler, J. B., Shaw, R. A., \& Browning, L. 1997, PASP, 109, 289

Kaler, J. B., \& Jacoby, G. H. 1990, ApJ, 362, 491

Kaler, J. B., Kwitter, K. B., Shaw, R. A., \& Browning, L. 1996, PASP, 108, 980

Kingsburgh, R. L., \& Barlow, M. J. 1994, MNRAS, 271, 257

Kwitter, K. B., \& Henry, R. B. C. 2001, ApJ, 562, 804

Maciel, W. J. 1984, A\&AS, 55, 253

Maciel, W. J., \& Quireza, C. 1999, A\&A, 345, 629

Maciel, W. J., Costa, R. D. D., \& Uchida, M. M. M. 2003, A\&A, 397, 667

Maciel, W. J., Lago, L. G., \& Costa, R. D. D. 2005, A\&A, 433, 127

Manchado, A., Guerrero, M. A., Stanghellini, L., \& Serra-Ricart, M. 1996, The IAC morphological catalog of northern galactic planetary nebulae, Publisher: La Laguna, Spain: Instituto de Astrofisica de Canarias (IAC), 1996, Foreword by Stuart R. Pottasch, ISBN: 8492180609,

Manchado, A., Villaver, E., Stanghellini, L., \& Guerrero, M. A. 2000, ASP Conf. Ser. 199: Asymmetrical Planetary Nebulae II: From Origins to Microstructures, 199, 17

Marigo, P. 2001, A\&A, 370, 194

Martin-Hernandez, N. L., Peeters, E., Morisset, C., Tielens, A. G. G. M., Cox, P., Roelfsema, P. R., Baluteau, J-P, Schaerer, D., Mathis, J. S., Damour, F., Churchwell, E., \& Kessler, M. F. 2002, A\&A, 381, 606

Martins, L. P., \& Viegas, S. M. M. 2000, A\&A, 361, 1121

Peimbert, M., \& Torres-Peimbert, S. 1983, IAU Symp. 103: Planetary Nebulae, 103, 233

Matteucci, F. 2003, The Chemical Evolution of the Galaxy. By Francesca Matteucci, Department of Astronomy, University of Trieste, Italy. Astrophysics and Space Science Library Volume 253 reprint Kluwer Academic Publishers, Dordrecht,

Perinotto, M., Morbidelli, L., \& Scatarzi, A. 2004, MNRAS, 349, 793 
Pilyugin, L. S., Ferrini, F., \& Shkvarun, R. V. 2003, A\&A, 401, 557

Shaw, R. A., de la Peña, M. D., Katsanis, R. M., \& Williams, R. E. 1998, in Astronomical Data Analysis Software and Systems VII, A.S.P. Conference Series, Vol. 145, R. Albrecht, R. N. Hook \& H. A. Bushouse, eds., p. 192

Schwarz, H. E., Corradi, R. L. M., \& Melnick, J. 1992, A\&AS, 96, 23

Stanghellini, L., Corradi, R. L. M., \& Schwarz, H. E. 1993, A\&A, 279, 674

Stanghellini, L., Blades, J. C., Osmer, S. J., Barlow, M. J., \& Liu, X.-W. 1999, ApJ, 510, 687

Stanghellini, L., Villaver, E., Manchado, A., \& Guerrero, M. A. 2002, ApJ, 576, 285

Stasinska, G. 2004, in Cosmochemistry, The melting pot of the elements. ed. C. Esteban, R. J. Garcia Lpez, A. Herrero, F. Sanchez, Cambridge University Press, p. 115

Trammell, S. R., Dinerstein, H. L., \& Goodrich, R. W. 1993, ApJ, 402, 249

van den Hoek, L. B., \& Groenewegen, M. A. T. 1997, A\&AS 123, 305

Whiteford, A. E. 1958, AJ, 63, 201 
Table 1. Abundances and distances

\begin{tabular}{lcrrrrr}
\hline \hline \multicolumn{1}{c}{ Name } & $\begin{array}{c}\mathrm{He} / \mathrm{H} \\
{\left[\times 10^{2}\right]}\end{array}$ & $\begin{array}{c}\mathrm{N} / \mathrm{H} \\
{\left[\times 10^{4}\right]}\end{array}$ & $\begin{array}{c}\mathrm{O} / \mathrm{H} \\
{\left[\times 10^{4}\right]}\end{array}$ & $\begin{array}{c}\mathrm{Ne} / \mathrm{H} \\
{\left[\times 10^{4}\right]}\end{array}$ & $\begin{array}{c}\mathrm{Ar} / \mathrm{H} \\
{\left[\times 10^{6}\right]}\end{array}$ & $\begin{array}{c}\mathrm{R} \\
{[\mathrm{kpc}]}\end{array}$ \\
\hline Round & & & & & & \\
\hline A 4 & 8.2 & 2.36 & 4.26 & $\ldots$ & $\ldots$ & $12.8^{2}$ \\
A 50 & 3.8 & 1.20 & 3.30 & $\ldots$ & $\ldots$ & $8.5^{1}$ \\
Cn 3-1 & 5.7 & 0.58 & 2.16 & 0.23 & 0.16 & $5.7^{2}$ \\
He 1-5 & 10.1 & 1.10 & 6.14 & 2.21 & $\ldots$ & $7.2^{5}$ \\
IC 4593 & 12.7 & 0.36 & 2.94 & 0.59 & $\ldots$ & $7.4^{5}$ \\
K 1-7 & 10.0 & 7.70 & 3.40 & 1.09 & $\ldots$ & $13.4^{2}$ \\
K 3-27 & 11.0 & $\ldots$ & 0.90 & 0.16 & 2.57 & $7.0^{1}$ \\
K 3-51 & 10.5 & $\ldots$ & 1.00 & 0.18 & $\ldots$ & $6.7^{1}$ \\
K 3-73 & 7.9 & 0.35 & 1.70 & 0.77 & $\ldots$ & $10.1^{3}$ \\
M 1-80 & 13.4 & 1.70 & 3.00 & 0.19 & 0.05 & $11.0^{2}$ \\
M 4-18 & 7.7 & 0.71 & 3.30 & $\ldots$ & $\ldots$ & $14.5^{2}$ \\
NGC 2242 & 11.7 & $\ldots$ & $\ldots$ & 0.04 & 0.82 & $\ldots$ \\
NGC 2438 & 10.3 & 0.85 & 4.50 & 1.03 & $\ldots$ & $9.4^{5}$ \\
NGC 3587 & 9.9 & 0.29 & 2.30 & 0.90 & $\ldots$ & $8.2^{5}$ \\
NGC 6879 & 11.4 & $\ldots$ & $\ldots$ & 0.69 & $\ldots$ & $7.4^{2}$ \\
NGC 6884 & 13.5 & 2.10 & 4.54 & 0.82 & 2.18 & $8.0^{5}$ \\
NGC 6894 & 11.1 & 4.49 & 12.35 & 5.16 & $\ldots$ & $7.6^{5}$ \\
Na 1 & $\ldots$ & $\ldots$ & $\ldots$ & 0.92 & 1.81 & $3.5^{4}$ \\
Vy 2-3 & 11.3 & 0.15 & 2.00 & 0.64 & 1.43 & $15.8^{3}$ \\
\hline Average & 10.0 & 1.71 & 3.61 & 0.98 & 1.29 & \\
\hline
\end{tabular}

\begin{tabular}{lcrrrrr} 
Elliptical & & & & & & \\
\hline A 2 & 7.2 & 1.00 & 3.38 & 1.18 & 6.40 & $10.6^{2}$ \\
A 43 & 10.8 & $\ldots$ & $\ldots$ & $\ldots$ & 4.05 & $6.8^{1}$ \\
A 70 & 9.4 & 1.78 & 3.20 & 0.77 & $\ldots$ & $6.5^{1}$ \\
CRL 618 & 4.9 & 0.56 & 3.40 & $\ldots$ & $\ldots$ & $11.4^{2}$ \\
Hu 1-1 & 10.9 & 1.50 & 5.80 & 1.96 & 0.30 & $10.8^{2}$
\end{tabular}


Table 1-Continued

\begin{tabular}{|c|c|c|c|c|c|c|}
\hline Name & $\begin{array}{l}\mathrm{He} / \mathrm{H} \\
{\left[\times 10^{2}\right]}\end{array}$ & $\begin{array}{c}\mathrm{N} / \mathrm{H} \\
{\left[\times 10^{4}\right]}\end{array}$ & $\begin{array}{c}\mathrm{O} / \mathrm{H} \\
{\left[\times 10^{4}\right]}\end{array}$ & $\begin{array}{l}\mathrm{Ne} / \mathrm{H} \\
{\left[\times 10^{4}\right]}\end{array}$ & $\begin{array}{c}\mathrm{Ar} / \mathrm{H} \\
{\left[\times 10^{6}\right]}\end{array}$ & $\begin{array}{c}\mathrm{R} \\
{[\mathrm{kpc}]}\end{array}$ \\
\hline IC 351 & 11.1 & $\ldots$ & $\ldots$ & 0.38 & 0.89 & $13.2^{2}$ \\
\hline IC 2003 & 11.6 & 1.90 & 4.00 & 0.80 & 1.09 & $10.6^{1}$ \\
\hline IC 2149 & 9.6 & 0.29 & 1.61 & 0.16 & 0.65 & $8.9^{5}$ \\
\hline J 320 & 11.4 & 0.32 & 1.51 & 0.46 & 0.62 & $13.7^{2}$ \\
\hline K 3-61 & $\ldots$ & 7.83 & 3.84 & 0.70 & 0.28 & $11.4^{2}$ \\
\hline K 3-92 & 11.0 & 1.45 & 6.41 & 2.61 & $\ldots$ & $13.4^{2}$ \\
\hline M 1-6 & 11.5 & 0.07 & 0.54 & $\ldots$ & 0.38 & $10.4^{1}$ \\
\hline M 1-64 & 11.3 & 1.70 & 2.60 & 1.10 & $\ldots$ & $7.5^{1}$ \\
\hline M 2-2 & 10.7 & 0.29 & 2.20 & 0.50 & 0.45 & $11.9^{2}$ \\
\hline M 2-44 & 29.1 & $\ldots$ & $\ldots$ & 0.05 & 1.63 & $\ldots$ \\
\hline M 2-50 & 3.9 & 1.10 & 2.30 & 0.55 & 1.37 & $13.6^{3}$ \\
\hline M 3-3 & 12.2 & 6.56 & 4.34 & 1.45 & 0.49 & $12.9^{2}$ \\
\hline Me 1-1 & 11.4 & 1.47 & 2.96 & 1.01 & 2.77 & $6.4^{1}$ \\
\hline NGC 40 & 9.3 & 0.79 & 3.30 & 0.26 & $\ldots$ & $8.6^{5}$ \\
\hline NGC 1514 & $\ldots$ & $\ldots$ & 1.10 & 0.37 & 1.32 & $8.7^{5}$ \\
\hline NGC 2022 & 13.4 & 0.88 & 4.50 & 1.06 & 2.03 & $9.5^{5}$ \\
\hline NGC 2392 & 11.2 & 1.14 & 2.36 & 0.51 & $\ldots$ & $8.6^{5}$ \\
\hline NGC 6210 & 11.4 & 0.50 & 3.60 & 0.90 & 0.66 & $6.9^{1}$ \\
\hline NGC 6543 & 11.2 & 0.92 & 4.59 & 1.39 & 0.65 & $8.1^{5}$ \\
\hline NGC 6720 & 11.7 & 1.80 & 6.00 & 1.70 & 0.32 & $7.7^{1}$ \\
\hline NGC 6741 & 23.1 & 5.90 & 4.40 & 1.02 & 2.61 & $6.7^{5}$ \\
\hline NGC 6826 & 10.6 & 0.23 & 2.52 & 0.52 & 0.30 & $8.0^{5}$ \\
\hline NGC 6853 & 12.1 & 2.20 & 7.00 & 2.24 & 1.88 & $7.9^{5}$ \\
\hline NGC 6905 & 12.8 & 0.80 & 5.00 & 1.45 & 0.85 & $7.3^{1}$ \\
\hline NGC 7008 & 12.7 & 0.47 & 1.47 & 0.77 & $\ldots$ & $8.1^{5}$ \\
\hline NGC 7048 & 13.5 & & $\ldots$ & $\ldots$ & $\ldots$ & $8.1^{5}$ \\
\hline NGC 7354 & 13.7 & 0.62 & 2.17 & 0.57 & 0.80 & $8.9^{5}$ \\
\hline NGC 7662 & 12.5 & 0.72 & 2.60 & 0.53 & 1.22 & $8.3^{5}$ \\
\hline PC 19 & 11.3 & 0.37 & 3.09 & $\ldots$ & $\ldots$ & $4.4^{2}$ \\
\hline
\end{tabular}


Table 1-Continued

\begin{tabular}{rccccccc}
\hline \hline Name & $\begin{array}{c}\mathrm{He} / \mathrm{H} \\
{\left[\times 10^{2}\right]}\end{array}$ & $\begin{array}{c}\mathrm{N} / \mathrm{H} \\
{\left[\times 10^{4}\right]}\end{array}$ & $\begin{array}{c}\mathrm{O} / \mathrm{H} \\
{\left[\times 10^{4}\right]}\end{array}$ & $\begin{array}{c}\mathrm{Ne} / \mathrm{H} \\
{\left[\times 10^{4}\right]}\end{array}$ & $\begin{array}{c}\mathrm{Ar} / \mathrm{H} \\
{\left[\times 10^{6}\right]}\end{array}$ & $\begin{array}{c}\mathrm{R} \\
{[\mathrm{kpc}]}\end{array}$ \\
\hline Vy 1-1 & $\ldots$ & 0.76 & 2.30 & 0.46 & 0.56 & $12.1^{2}$ \\
Vy 1-2 & 17.1 & 0.79 & 2.90 & 0.65 & 0.69 & $7.6^{3}$ \\
\hline Average & 12.0 & 1.51 & 3.34 & 0.91 & 1.31 & \\
\hline
\end{tabular}

\begin{tabular}{lcrrrrr} 
Bipolar & \multicolumn{7}{c}{$\ldots$} & & & \\
\hline K 3-46 & 10.3 & $\ldots$ & $\ldots$ & $\ldots$ & $\ldots$ & $7.5^{1}$ \\
K 3-94 & 13.7 & $\ldots$ & $\ldots$ & $\ldots$ & $\ldots$ & $13.7^{2}$ \\
K 4-55 & 14.0 & 10.00 & 2.40 & 0.54 & 3.30 & $8.2^{1}$ \\
M 1-57 & 12.8 & 1.79 & 4.62 & $\ldots$ & $\ldots$ & $7.5^{3}$ \\
M 1-59 & 27.2 & $\ldots$ & $\ldots$ & 1.18 & $\ldots$ & $6.9^{5}$ \\
M 1-75 & 21.0 & 23.00 & 5.30 & 1.57 & 3.88 & $7.4^{1}$ \\
M 2-46 & 8.1 & 2.17 & 1.58 & $\ldots$ & $\ldots$ & $5.8^{4}$ \\
M 2-52 & 17.2 & 5.26 & 3.95 & $\ldots$ & 1.03 & $10.0^{2}$ \\
M 3-28 & 11.4 & 8.52 & 5.35 & 1.11 & $\ldots$ & $3.9^{4}$ \\
NGC 2346 & 13.5 & 2.00 & 3.20 & 0.99 & 0.35 & $8.9^{5}$ \\
NGC 6778 & 16.2 & 2.16 & 1.29 & 0.42 & 0.24 & $5.7^{2}$ \\
NGC 6881 & 12.4 & 2.49 & 4.02 & 1.18 & 0.98 & $7.7^{1}$ \\
NGC 7026 & 12.4 & 5.40 & 4.70 & 1.13 & 0.64 & $8.2^{5}$ \\
NGC 7027 & 16.3 & 2.70 & 2.70 & 0.47 & 0.94 & $8.0^{5}$ \\
Pe 1-17 & 17.6 & 6.10 & 9.46 & $\ldots$ & $\ldots$ & $3.4^{2}$ \\
\hline Average & 14.9 & 5.97 & 4.05 & 0.95 & 1.42 & \\
\hline
\end{tabular}

Bipolar Core

\begin{tabular}{lcrrrrr}
\hline He 1-6 & 12.8 & $\ldots$ & $\ldots$ & $\ldots$ & $\ldots$ & $7.3^{1}$ \\
Hu 1-2 & 18.5 & 1.90 & 1.90 & 0.52 & 0.86 & $8.1^{5}$ \\
M 1-7 & 9.7 & 1.50 & 2.90 & 0.94 & 0.24 & $13.8^{2}$ \\
M 1-79 & 13.2 & 1.77 & 1.59 & $\ldots$ & $\ldots$ & $8.4^{5}$
\end{tabular}


Table 1-Continued

\begin{tabular}{lrrrrrr}
\hline \hline Name & $\begin{array}{c}\mathrm{He} / \mathrm{H} \\
{\left[\times 10^{2}\right]}\end{array}$ & $\begin{array}{c}\mathrm{N} / \mathrm{H} \\
{\left[\times 10^{4}\right]}\end{array}$ & $\begin{array}{c}\mathrm{O} / \mathrm{H} \\
{\left[\times 10^{4}\right]}\end{array}$ & $\begin{array}{c}\mathrm{Ne} / \mathrm{H} \\
{\left[\times 10^{4}\right]}\end{array}$ & $\begin{array}{c}\mathrm{Ar} / \mathrm{H} \\
{\left[\times 10^{6}\right]}\end{array}$ & $\begin{array}{c}\mathrm{R} \\
{[\mathrm{kpc}]}\end{array}$ \\
\hline M 2-51 & 14.8 & 1.98 & 3.80 & $\ldots$ & $\ldots$ & $8.6^{1}$ \\
M 2-53 & 14.9 & 1.63 & 4.43 & 1.93 & 0.56 & $9.6^{1}$ \\
M 2-55 & 14.0 & 2.65 & 3.25 & 1.08 & $\ldots$ & $9.2^{1}$ \\
NGC 6058 & 12.4 & $\ldots$ & $\ldots$ & $\ldots$ & $\ldots$ & $7.3^{1}$ \\
NGC 6804 & 15.0 & $\ldots$ & 5.34 & 2.35 & 0.75 & $7.0^{5}$ \\
\hline Average & 13.9 & 1.91 & 3.32 & 1.36 & 6.03 &
\end{tabular}

$1 \overline{\Delta \mathrm{R} / \mathrm{R}<0.2 ;{ }^{2} 0.2<\Delta \mathrm{R} / \mathrm{R}<0.4 ;{ }^{3} 0.4<\Delta \mathrm{R} / \mathrm{R}<0.6 ;{ }^{4} \Delta \mathrm{R} / \mathrm{R}>0.6}$

${ }^{5}$ Individual distances from Acker et al. 1992 
Table 2. Oxygen abundance comparison

\begin{tabular}{lrrr}
\hline \hline $\begin{array}{c}\text { O } / \mathrm{H}> \pm \sigma \\
{\left[\times 10^{4}\right]}\end{array}$ & $\begin{array}{c}\text { Our sample } \\
67 \mathrm{PNe}\end{array}$ & $\begin{array}{c}\text { PMS04 sample } \\
27 \mathrm{PNe}\end{array}$ & $\begin{array}{c}\text { HKB04 sample } \\
17 \mathrm{PNe}\end{array}$ \\
& & & \\
\hline This paper & $3.5 \pm 2.0$ & $3.9 \pm 2.2$ & $3.7 \pm 1.3$ \\
Other studies & $\ldots$ & $4.3 \pm 1.9$ & $5.6 \pm 1.7$ \\
\hline
\end{tabular}

Table 3. Gradients

\begin{tabular}{lrrrr}
\hline \hline Ratio & $\begin{array}{c}\text { This work } \\
{\left[\mathrm{dex} \mathrm{kpc}^{-1}\right]}\end{array}$ & $\begin{array}{c}\text { HKB04 } \\
{\left[\mathrm{dex} \mathrm{kpc}^{-1}\right]}\end{array}$ & $\begin{array}{c}\text { MV00 } \\
{\left[\mathrm{dex} \mathrm{kpc}^{-1}\right]}\end{array}$ & $\begin{array}{c}\text { MQ99 } \\
{\left[\mathrm{dex} \mathrm{kpc}^{-1}\right]}\end{array}$ \\
\hline $\mathrm{O} / \mathrm{H}$ & -0.01 & -0.04 & -0.05 & -0.06 \\
$\mathrm{Ne} / \mathrm{H}$ & -0.01 & -0.04 & -0.07 & -0.04 \\
$\mathrm{Ar} / \mathrm{H}$ & -0.05 & -0.03 & $\ldots$ & -0.05 \\
\hline
\end{tabular}




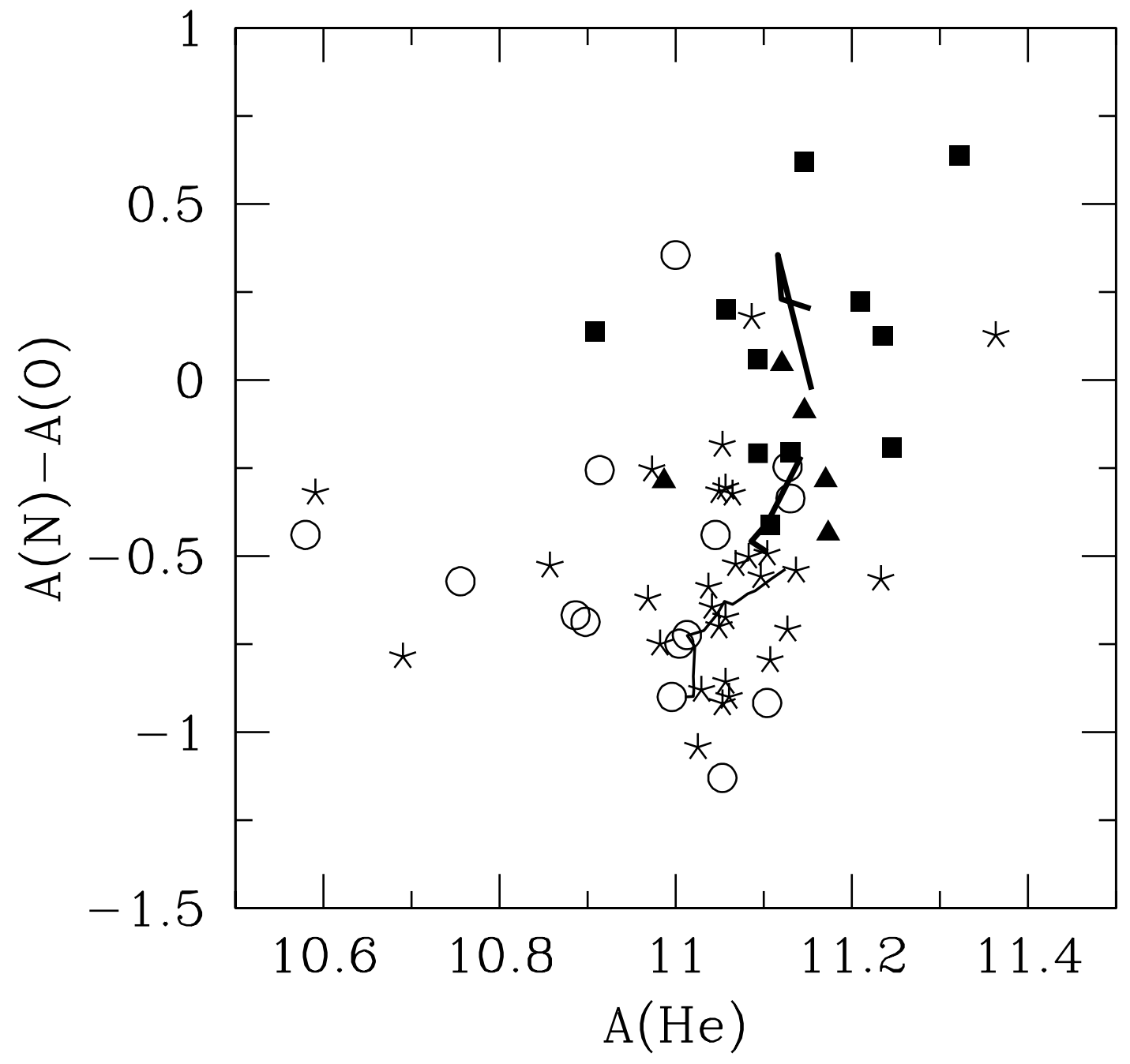

Fig. 1. $-\mathrm{A}(\mathrm{N})-\mathrm{A}(\mathrm{O})$, or $\log (\mathrm{N} / \mathrm{O})$, versus $\mathrm{A}(\mathrm{He})$, or $\log (\mathrm{He} / \mathrm{H})+12$. Symbols indicate morphology types: Round (open circles), Elliptical (asterisks), Bipolar Core (triangles) and Bipolar (squares). Solid lines: galactic models by Marigo (2001), thin line connect models with $\mathrm{M}_{\mathrm{TO}}<3 \mathrm{M}_{\odot}$ and $\alpha$ - the mixing-length parameter- $=1.68$, thick line connects models 


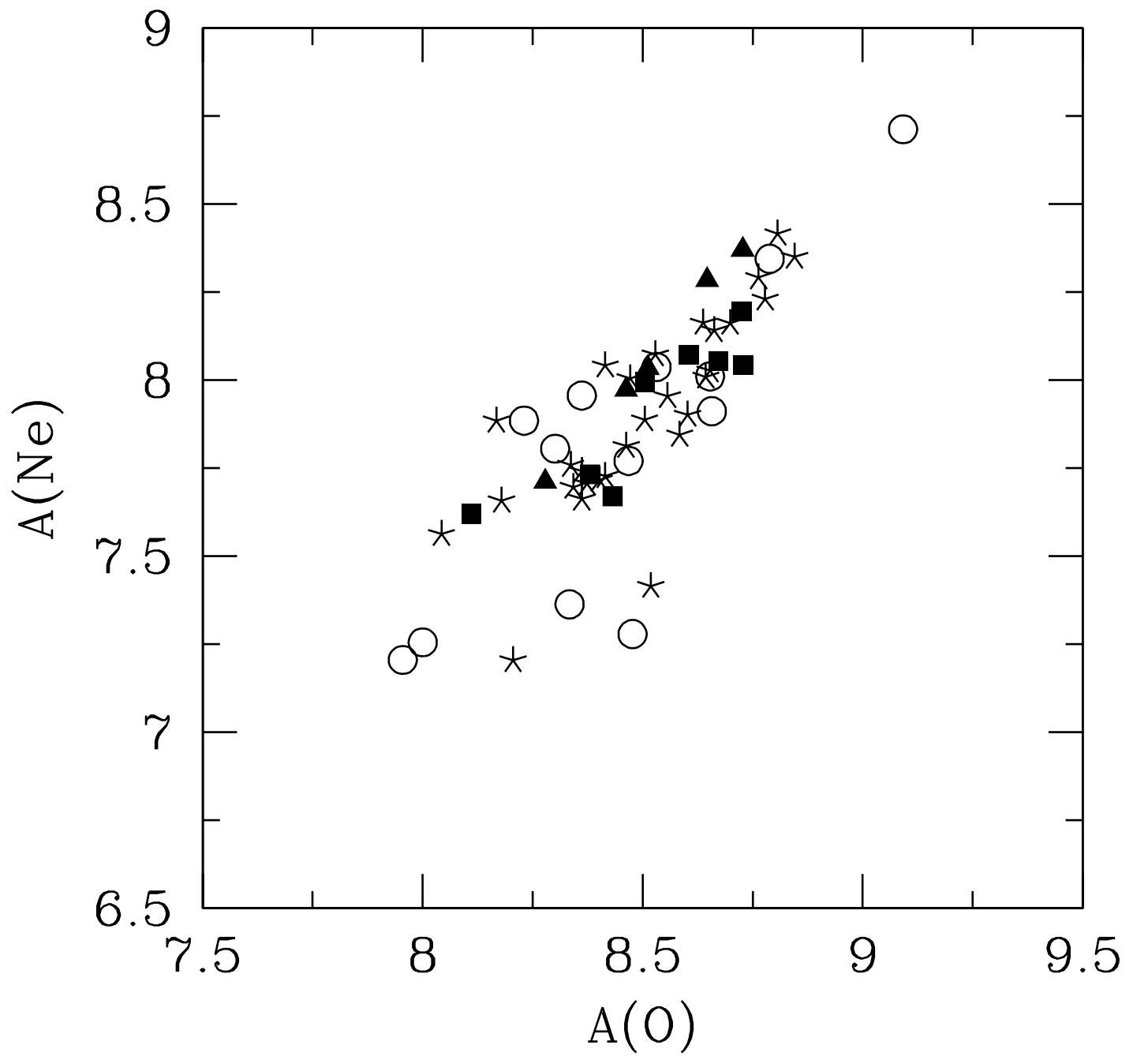

Fig. 2.- $\mathrm{A}(\mathrm{Ne})$ versus $\mathrm{A}(\mathrm{O})$. Symbols are as in Figure 1. 


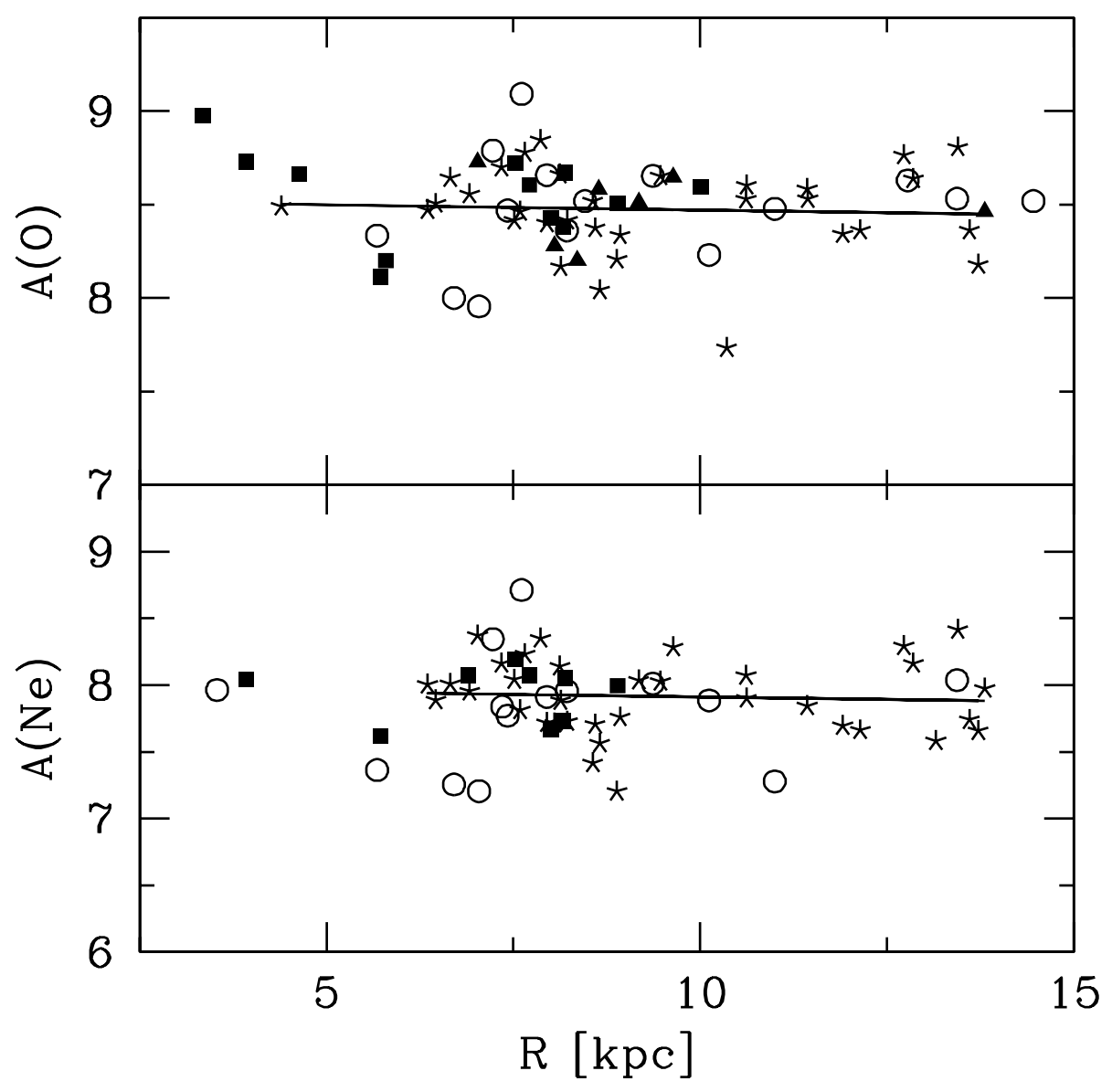

Fig. 3.- Oxygen (top) and neon (bottom) abundances, given as $\log (\mathrm{X} / \mathrm{H})+12$, versus galactocentric distances. Symbols are as in Figure 1. The lines represent least squares fits, their inclination given in Table 3, and they include E and BC PNe only. 